OPEN ACCESS

Edited by:

Huaiyang Yuan,

Utrecht University, Netherlands

Reviewed by:

Farhad Sattari,

University of Mohaghegh Ardabili, Iran

Rafael A. Molina,

Consejo Superior de Investigaciones Cientificas (CSIC), Spain

*Correspondence: M. H. Qin

qinmh@scnu.edu.cn

Specialty section:

This article was submitted to Condensed Matter Physics, a section of the journal

Frontiers in Physics

Received: 07 August 2021 Accepted: 27 September 2021 Published: 03 November 2021

Citation:

Guan SH, Yang Y, Jin Z, Liu TT, Liu Y and Qin MH (2021) Suppression of

Skyrmion Hall Motion in Antiferromagnets Driven by Circularly

Polarized Spin Waves.

Front. Phys. 9:754869.

doi: 10.3389/fphy.2021.754869

\section{Suppression of Skyrmion Hall Motion in Antiferromagnets Driven by Circularly Polarized Spin Waves}

\author{
S. H. Guan, Y. Yang, Z. Jin, T. T. Liu, Y. Liu and M. H. Qin*
}

Guangdong Provincial Key Laboratory of Quantum Engineering and Quantum Materials and Institute for Advanced Materials, South China Academy of Advanced Optoelectronics, South China Normal University, Guangzhou, China

An investigation of spin waves interacting with antiferromagnetic spin textures is meaningful for future spintronic and magnonic-based memory and logic applications. In this work, we numerically study the skyrmion dynamics driven by circularly polarized spin waves in antiferromagnets and propose a method of suppressing the Hall motion. It is demonstrated that the application of two circularly polarized spin waves with opposite chirality allows the skyrmion motion straightly along the intersection line of the two spin wave sources. The skyrmion speed depending on these parameters of the spin waves and system is estimated, and a comparison with other methods is provided. Furthermore, two depinning behaviors of the skyrmion related to the strengths of the defect are also observed in the simulations. Thus, the proposed method could be used in precisely modulating the skyrmion dynamics, contributing to skyrmion-based memory device design.

Keywords: antiferromagnetic skyrmion, spin waves, dynamics, Hall motion, speed

\section{INTRODUCTION}

Skyrmion, a topologically protected particle-like magnetic structure, is considered as a promising candidate for spintronic applications like racetrack memories due to some of its attractive features including nanoscale in size, low critical driving current, and high stability [1-6]. Skyrmion dynamics driven by spin-polarized current in ferromagnets has been widely studied, and the so-called skyrmion Hall motion induced by the Magnus force is reported. In this case, the skyrmion moves deviating from the direction of the driving force and could be restricted or even annihilated by element edges of related devices [7-10]. Thus, the Hall motion brings a great challenge to the skyrmion-based applications.

Recently, antiferromagnetic (AFM) skyrmion has been predicted theoretically [11] and observed experimentally at room temperatures in synthetic antiferromagnets [12]. AFM skyrmion is comprised of two coupled spin configurations with opposite topological numbers, and two Magnus forces respectively acting on two sub-lattices under spin-polarized current perfectly cancel with each other, allowing the motion straightly along the driving force [13-16]. Moreover, compared with ferromagnetic skyrmion, AFM skyrmion has more attractive physical properties. For instance, AFM skyrmion is insensitive to external magnetic field [17, 18], and its speed is much larger than ferromagnetic skyrmion under a same driving current density [15]. Importantly, AFM materials are abundant in nature, which include metals comprised Mn-based alloys, insulators, semiconductors, and semimetals [19]. All these merits of AFM skyrmions contribute to the rapid development of AFM spintronics. 
Most recently, spin wave driven skyrmion motion draws a lot of attention due to some advantages such as low dissipation and producing less heat [20-22]. In ferromagnets, the spin wave is only right-circularly polarized and drives the skyrmion motion towards the spin wave source in the presence of a Hall motion related to its topological charge, which can be understood from the momentum conservation [23]. In antiferromagnets, however, the spin wave can be fully polarized, providing another degree of freedom in modulating the skyrmion dynamics [24]. For AFM system with uniaxial anisotropy, the spin wave is generally polarized [40], and the scattering of the spin wave is related to its polarization direction, resulting in interesting and complex skyrmion dynamics.

For example, when AFM skyrmion is driven by chiral or isospin charged spin wave with circular or elliptical polarizations, a magnonic topological spin Hall effect is produced resulting from the symmetry breaking of the magnon trajectory [25]. Thus, the skyrmion Hall motion could be also driven by the spin wave even in AFM systems. Interestingly, the scattering direction of the magnon and the deflection direction of skyrmion are determined by the chirality of the spin wave, allowing the skyrmions work as spin splitters in AFM magnon devices [26]. However, skyrmion Hall motion is detrimental for data propagation in future skyrmion-based devices, as discussed above. It is predicted that the linearly polarized spin wave with particular polarization directions drives the AFM skyrmion straightly along its propagation direction without any Hall motion [27], while the strict limitation of the polarization directions affects stable application. Furthermore, the skyrmion speed driven by linearly polarized spin waves is rather low compared with one driven by circularly polarized spin wave. Thus, there is still an urgent need in suppressing the skyrmion Hall motion driven by spin waves in antiferromagnets, considering these particular merits in future magnonic and spintronic device design.

In this work, we propose a method of suppressing skyrmion Hall motion by injecting two kinds of spin waves with opposite chirality in antiferromagnets. The application of the two circularly polarized spin waves drives the skyrmion motion straightly along the intersection line. The skyrmion speed depending on these parameters of the spin waves and system is numerically simulated, and a comparison with those driven by other methods is provided and briefly discussed. Furthermore, the depinning behaviors of the skyrmion from the defects are also investigated.

\section{MODEL AND METHOD}

We consider a two-dimensional AFM Heisenberg model in the $x y$ plane with the model Hamiltonian,

$$
H=J \sum_{\langle i, j\rangle} \mathbf{m}_{i} \cdot \mathbf{m}_{j}+\sum_{\langle i, j\rangle} \mathbf{D} \cdot\left(\mathbf{m}_{i} \times \mathbf{m}_{j}\right)-K \sum_{i}\left(\mathbf{m}_{i}^{z}\right)^{2},
$$

where the first term is the AFM exchange interaction between the nearest neighbors with $J>0, \mathbf{m}_{i}$ is the local magnetic moment at site $i, \mathbf{m}_{i}=-\mathbf{S}_{i} / \hbar$ with the local spin $\mathbf{S}_{i}$, and the reduced Plank constant $\hbar$, the second term is the Dzyaloshinskii-Moriya interaction (DMI) between the nearest neighbors with the vector $\mathbf{D}=D \mathbf{e}_{i j}$, the third term is the perpendicular magnetic

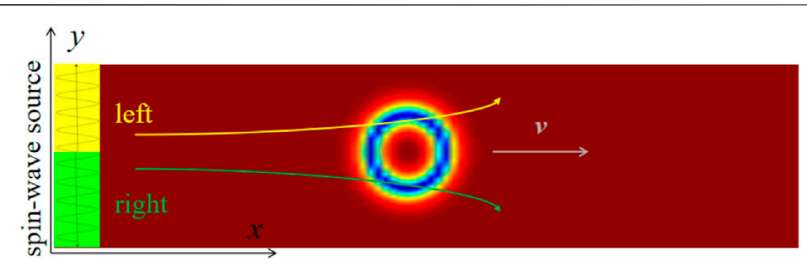

FIGURE 1 | The model of AFM skyrmion driven by double circularly polarized spin waves. The yellow and green regions represent the source of left-handed and right-handed circularly polarized spin waves, respectively. The yellow and green arrows indicate the scattering direction of the spin waves, and the gray arrow indicates the driving direction of the skyrmion.

anisotropy with the easy $z$-axis and magnitude $K$. Considering the canted AFM sample $\mathrm{KMnF}_{3}$, which has been widely used in the study of spin wave propagation theoretically and experimentally [28-30], the parameters are chosen to be $J=1.0 \times 10^{-21} \mathrm{~J}, D / J=$ 0.14 , and $K / J=0.03$ [31]. In addition, other AFM materials with stable existence of skyrmion and spin wave propagation such as two AFM coupled layers of YIG [26] and Co/Pt bilayer [12, 32] could also be applicable to our simulation.

Subsequently, the time-dependent magnetization dynamics are investigated using the Landau-Lifshitz-Gilbert (LLG) equation,

$$
\frac{d \mathbf{m}_{i}}{d t}=-\gamma \mathbf{m}_{i} \times\left(\mathbf{H}_{i}^{e f f}+\mathbf{h}\right)+\alpha \mathbf{m}_{i} \times \frac{d \mathbf{m}_{i}}{d t}
$$

where $\mathbf{H}_{i}^{e f f}=-\left(1 / \mu_{0}\right) \partial H / \partial \mathbf{m}_{i}$ is the effective field. We set the lattice constant $a=1.0 \times 10^{-9} \mathrm{~m}$, the gyromagnetic ratio $\gamma=-2.211 \times$ $10^{5} \mathrm{~m} /(\mathrm{a} . \mathrm{s})$ [13] and the Gilbert damping coefficient $\alpha=0.005$. h is the AC magnetic field used to generate stable spin wave, $\mathbf{h}_{\mathrm{LH}} /$ $\mathbf{h}_{\mathrm{RH}}=h_{0}(\sin (\omega t) \mathbf{i} \pm \cos (\omega t) \mathbf{j})$ corresponds to left-/right-circularly polarized spin wave, and $\mathbf{h}_{x} / \mathbf{h}_{y}=h_{0} \sin (\omega t) \mathbf{j} / \mathbf{i}$ corresponds to the $x / y$ linearly polarized spin wave with the magnitude $h_{0}$. We use the fourth-order Runge-Kutta method for numerical simulation on a system of $200 \times 100$ lattices. In our simulation, we set time step $\Delta t=0.01, J=1$, then a reduced time corresponds to the real time $t=1.0 \times 10^{-13} \mathrm{~s}$. Moreover, $\omega=1$ corresponds to $1.6 \mathrm{THz}$, and $h_{0}=1$ corresponds to 1.58 Tesla.

As depicted in Figure 1, the spin wave sources are set at the left side of the system, and the yellow part and green part represent the left-handed and right-handed spin wave sources, respectively. Unless stated elsewhere, we take the parameters $h_{0}=800 \mathrm{mT}$ and $\omega=2 \mathrm{THz}$ and use absorbing boundary on the right side of the system to avoid reflection of spin wave. Furthermore, the parameters of the two kinds of spin waves are set to be the same, and the balance position of the skyrmion is in the intersection line between the two sources. It is worth noting that the generating of the two kinds of spin waves with opposite chirality in the two halves of the system does not consume more energy than the injecting of single handed spin wave in the whole system.

\section{RESULTS AND DISCUSSION}

To some extent, spin wave scattering is similar to the classical motion of a particle subjecting to an effective magnetic field from 

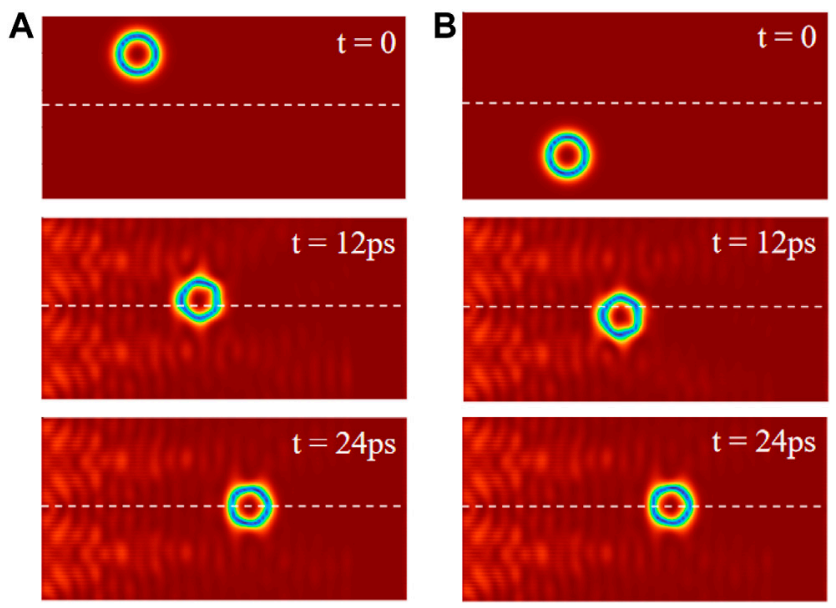

FIGURE 2 | The motion of the AFM skyrmion with different initial positions at (A) upside and (B) bottom side. The white dashed line indicates the intersection of the two spin wave sources.

the skyrmion [26]. Generally, due to their opposite effective charges, the left-handed and right-handed spin waves are scattered by the skyrmion towards the up side and down side, respectively, as depicted in Figure 1. Thus, the momentum exchanges between spin waves and the skyrmions along the longitudinal $y$-direction are well canceled out, and the skyrmion Hall motion could be suppressed.

This argument has been confirmed in our simulations. Figure 2A shows the evolution of the skyrmion position. The AFM skyrmion is initially at the upper side in the absence of the spin wave (up part). When the spin waves are injected, the skyrmion is driven to the right side. In this case, the left-handed spin wave is scattered to the upside and drives the skyrmion downward due to the momentum conservation. Thus, the skyrmion is quickly relaxed to its equilibrium position $y=50$ at $t=12 \mathrm{ps}$, as shown in the middle part of Figure 2A. Subsequently, the forces along the $y$-direction from the left-handed and right-handed spin waves acting on the skyrmion are well canceled out, and the skyrmion straightly moves along the $x$ direction. More importantly, the suppression of the Hall motion is not affected by the initial position of the skyrmion. Figure 2B shows the motion of the skyrmion initially at the bottom side of the system. Attributing to the effect of the righthanded spin wave, the skyrmion is also relaxed to the equilibrium position. Thus, this method is rather efficient in the suppression of the skyrmion Hall motion in antiferromagnets.

The simulated skyrmion position as a function of time is presented in Figure 3. On one hand, the skyrmion is quickly relaxed to the equilibrium $y$-position at $y=50$ (dashed line in Figure 3A) as discussed above, although a weak oscillating behavior is noticeable. On the other hand, the evolutions of the $x$-position for the two cases are almost the same, as shown in Figure 3B. It is noted that the injected spin waves are unavoidable dissipated as it propagates, contributing to the decrease of the skyrmion speed (slope of the position curve).

Subsequently, we investigate the dependence of the speed of the skyrmion on several parameters and present the simulated results in Figure 4. Here, the speed is estimated from the average one in the region $x=(110,130)$. The calculated velocity $v_{x^{-}}$ Double as a function of $\omega$ for $h_{0}=600 \mathrm{mT}$ is shown with red squares in Figure 4A. Two frequencies at $\omega=1.6 \mathrm{THz}$ and $2.2 \mathrm{THz}$ are observed with local maximum values $v_{x} \sim 450 \mathrm{~m} / \mathrm{s}$, which is comparable to the current driven one. The speed of the skyrmion driven by the $y$ linearly polarized spin wave $v_{x}$-Linearly is also estimated to be $v_{x} \sim 200 \mathrm{~m} / \mathrm{s}$, which is rather stable for various $\omega$ but much smaller than the maximum value driven by the double circularly polarized spin waves. To have a direction comparison, the speeds along the $x$ direction $v_{x}$ and $y$-direction $v_{y}$ of the skyrmion driven by right-handed spin wave are also presented. Two local maximum $v_{x} \sim 550 \mathrm{~m} / \mathrm{s}$ are estimated at $\omega=1.7 \mathrm{THz}$ and $2.0 \mathrm{THz}$, and the maximum $v_{y} \sim 850 \mathrm{~m} / \mathrm{s}$ is estimated at $\omega=$ $2.1 \mathrm{THz}$. However, in all investigated $\omega$ regions, $v_{y}>v_{x}$ is estimated, demonstrating the strong skyrmion Hall effect. Thus, particular $\omega$ could be chosen in the proposed method to obtain large speed in the absence of skyrmion Hall motion.

The magnitude of the spin wave is mainly controlled by $h_{0}$. Thus, with the increase of $h_{0}$, the speed of the skyrmion is increased, as shown in Figure $\mathbf{4 B}$, which presents the estimated speeds of the skyrmion as functions of magnitude driven by various methods at $\omega=2 \mathrm{THz}$. For $h_{0}<750 \mathrm{mT}$, all the estimated speeds increase appropriately with $h_{0}^{2}$ raised from the energy transfer between the spin wave and skyrmion, noting that the energy of the spin wave linearly increases with $h_{0}^{2}$. However, with the further increase of $h_{0}$, the skyrmion speed driven by the double circularly polarized spin waves increases much slowly. In some extent, the interference between the two kinds of spin waves is strengthened, strongly limiting the increase of the skyrmion speed.

Furthermore, the skyrmion radius depending on several parameters of the system such as the DMI and anisotropy also affects the skyrmion speed. In Figure 4C, the simulated speeds as functions of $D$ driven by various methods for $\omega=$ $2.0 \mathrm{THz}$ and $h_{0}=600 \mathrm{mT}$ are presented. Moreover, the calculated skyrmion radii are also given to help one to understand the results easier. The same as our earlier theoretical calculations, the radius is increased with the increase of $D[33,34]$. On one hand, the interacting area between the spin wave and skyrmion is enlarged [35], resulting in the increase of interacting force and skyrmion speed. On the other hand, the mass of the skyrmion is also increased with the increase of the radius [36], decreasing the skyrmion speed. As a result, the competition between the above two factors determines the speed of the skyrmion. For the skyrmion driven by the linearly polarized spin wave, the speed monotonously decreases with the increase of $D$, demonstrating the dominant effect of the second factor. For the skyrmion driven by double and single circularly polarized spin waves, the oscillating decrease of $v_{x}$ is observed due to the competition between the two factors. Moreover, the two simulated $v_{x}$ are almost the same for $D>0.145$, attributing to the increase of the component from the circularly polarized spin waves in the driving force.

Figure 4D shows the dependence of $v_{x}$ on the distance between the two spin wave sources for $\omega=2.0 \mathrm{THz}$ and 


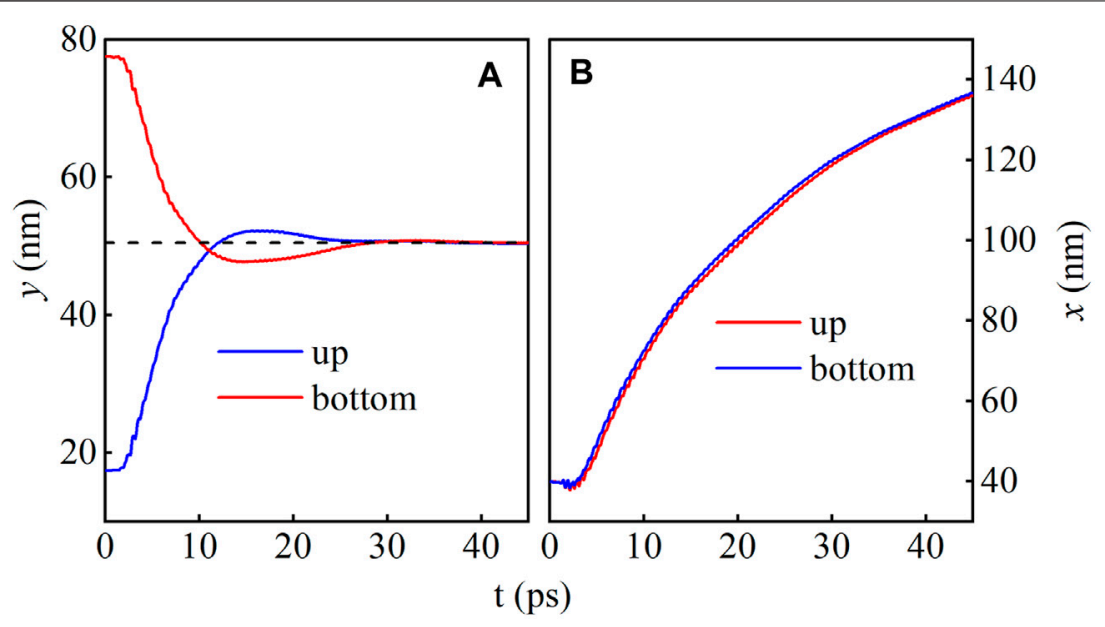

FIGURE 3 | The evolution of the (A) $y$ and (B) $x$ coordinates of the AFM skyrmions. The black dashed line represents the intersection of the spin wave sources.

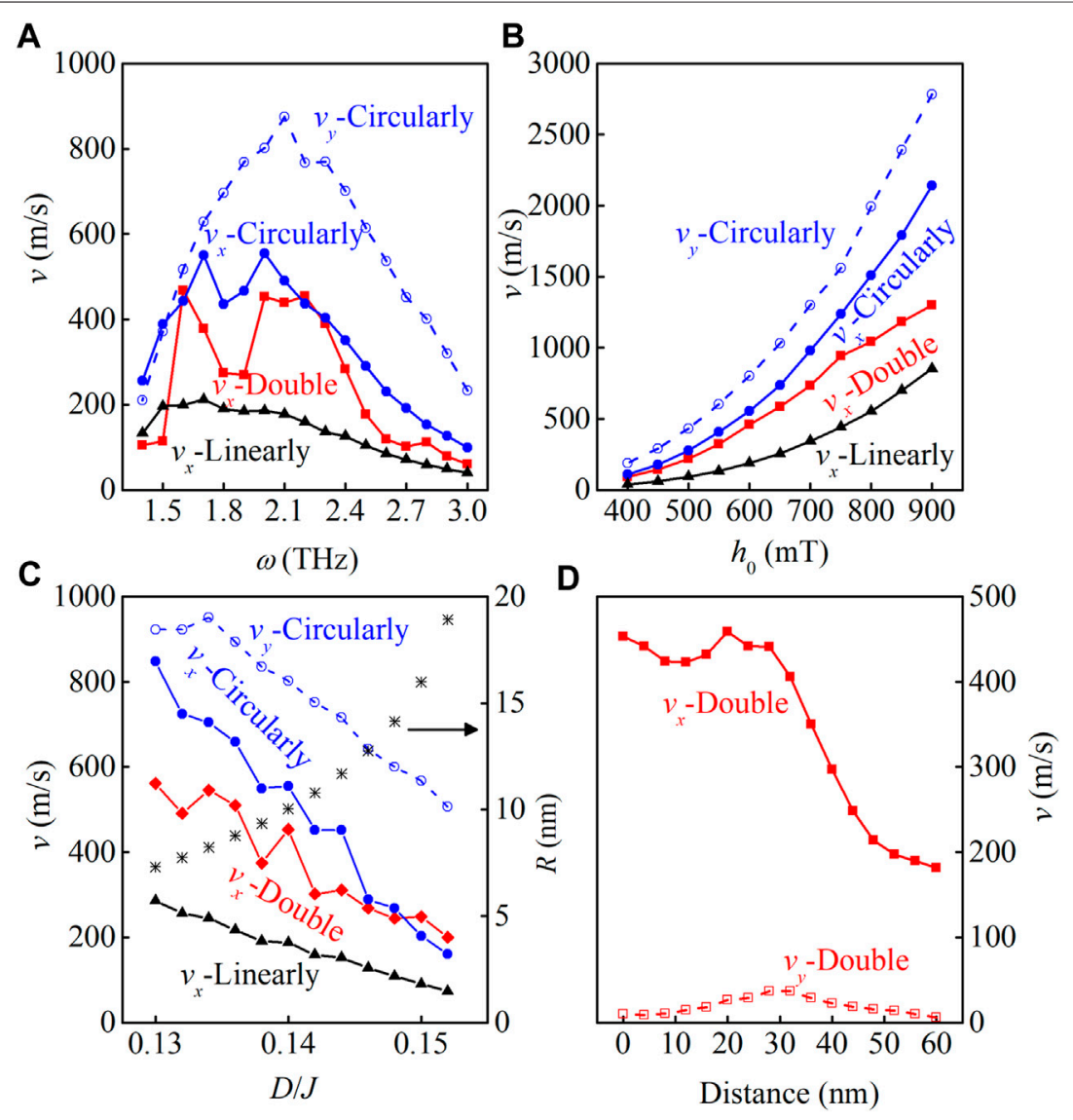

FIGURE 4 | The simulated speeds of the skyrmion driven by the double circularly polarized spin waves $v_{x}$-Double, by the $y$ linearly polarized spin waves $v_{x}$-Linearly, by the right-handed polarized spin waves $v_{x}$-Circularly and $v_{y}$-Circularly as functions of (A) $\omega$ for $h_{0}=600 \mathrm{mT}$, (B) $h_{0}$ for $\omega=2 \mathrm{THz}$, and (C) $D$ for $h_{0}=600 \mathrm{mT}$ and $\omega=$ $2 \mathrm{THz}$. (D) The simulated $v_{x}$ and $v_{y}$ as function of the distance between the two spin wave sources for $h_{0}=600 \mathrm{mT}$ and $\omega=2 \mathrm{TH}$. The simulated skyrmion radii are also presented in (C). 
A
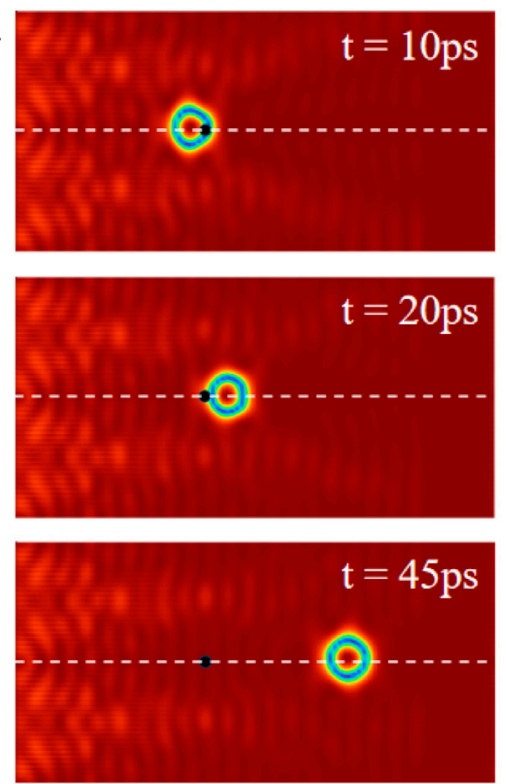

B
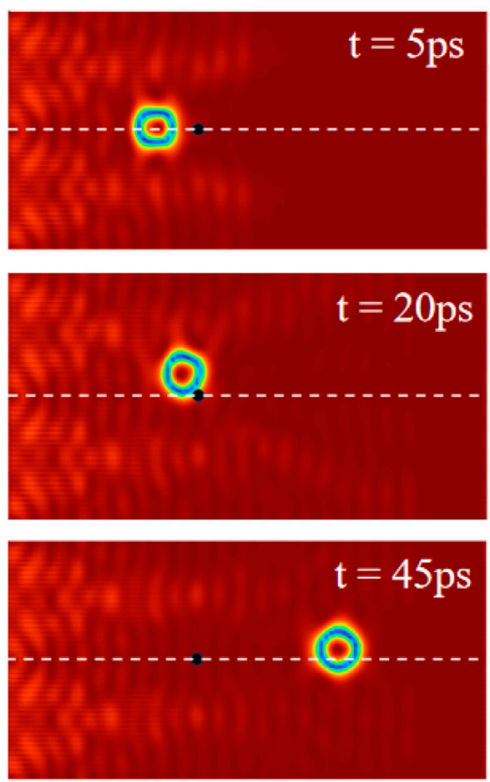

FIGURE 5 | The AFM skyrmion motion in the presence of the defect for $\mathbf{( A )} A / J=0.05$ and $\mathbf{( B )} A / J=0.15$. The black dots are the defects, and the white dashed lines represent the intersection of the two spin wave sources.

A

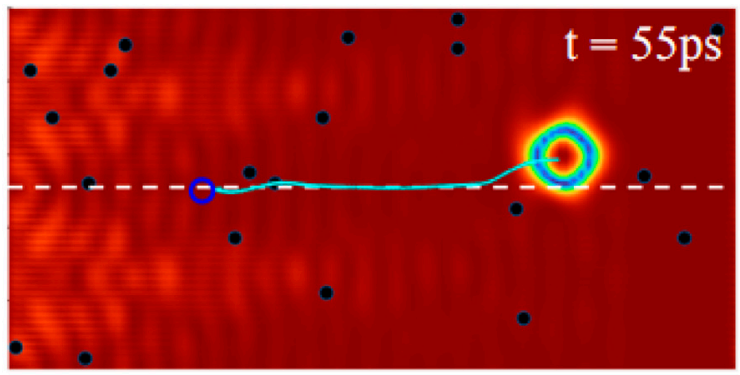

B

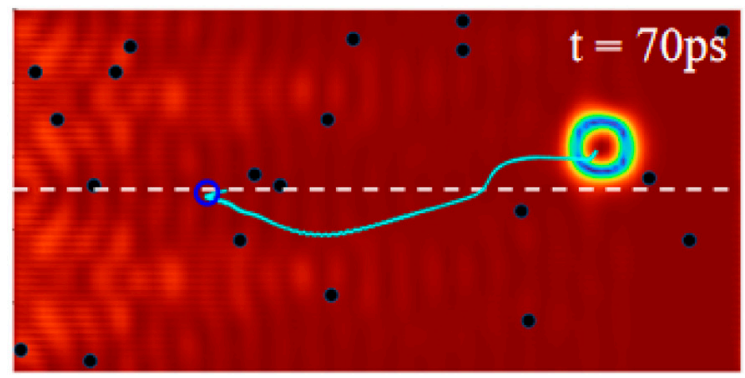

FIGURE 6 | The AFM skyrmion motion trajectories (green curves) with defect concentration $0.1 \%$ for (A) $A / J=0.05$ and (B) $A / J=0.15$, where the initial positions of skyrmion are represented by the empty blue circles.

$h_{0}=600 \mathrm{mT}$. It is clearly shown that in a rather large distance range $0-30 \mathrm{~nm}$, a rather stable speed $v_{x} \sim 500 \mathrm{~m} / \mathrm{s}$ is obtained. Thus, the skyrmion dynamics is hardly affected by increasing the distance between the two spin wave sources due to the spreading of the spin waves, allowing one to choose proper distance between the two spin wave sources to save energy. Moreover, $v_{y}$ is rather small in the whole distance range, indicating again the well suppression of the skyrmion Hall motion.

The depinning behavior of skyrmion is a crucial issue considering the fact that there are inevitable defects in real materials. At last, we investigate the depinning behavior from the defect which is set in the intersection line, as shown in Figure 5. Here, additional anisotropy $A\left(\mathrm{~m}_{i}^{z}\right)^{2}$ is used to describe the defect at site $i$, and we take $D / J=0.13$. Figure $5 \mathrm{~A}$ shows the trajectory of the skyrmion for $A / J=0.05$, $\omega=2 \mathrm{THz}$, and $h_{0}=800 \mathrm{mT}$. It is clearly shown that the skyrmion can pass through the defect directly. However, the energy barrier is high for large $A$, prohibiting the skyrmion go straightly through the defect [3]. Interestingly, the skyrmion can bypass the defect and then return to its presupposed orbit, as shown in Figure 5B, which presents the motion of the skyrmion for $A / J=0.15$. More interestingly, the depinning effect still works in the system with randomly distributed defects for a high concentration $0.1 \%$, as demonstrated in Figures $6 \mathbf{A}, \mathbf{B}$, where presents the skyrmion trajectory (green curve) for $A / J=0.05$ and $A / J=0.15$, respectively. Similarly, the skyrmions pass through the weak defects for $A / J=0.05$ directly, while bypassing the strong defects for $A / J=0.15$. Thus, the proposed method could be used to precisely drive the skyrmion motion along designed tracks.

Most recently, the AFM skyrmions have been experimentally observed in antiferromagnets with synthetic structures and three sub-lattices [37], which could be used to check our simulations. The generation of the spin wave could be realized through applying an oscillating magnetic field with intensity $h_{0}=400-800 \mathrm{mT}$ and frequency $\omega \sim 2 \mathrm{THz}$ [38], or by injecting spin current with intended polarizations $[39,40]$. 


\section{SUMMARY}

In summary, we propose a method to suppress the skyrmion Hall motion driven by circularly polarized spin waves in antiferromagnets. The application of the two circularly polarized spin waves drives the skyrmion motion straightly along the intersection line of the two spin wave sources. The skyrmion speed depending on these parameters of the spin waves and system is numerically simulated, and a comparison with those driven by other methods is provided. At last, two depinning behaviors of the skyrmion related to the strengths of the defect are discussed. Thus, the proposed method could be used in precisely modulating the skyrmion dynamics.

\section{DATA AVAILABILITY STATEMENT}

The raw data supporting the conclusions of this article will be made available by the authors, without undue reservation.

\section{REFERENCES}

1. Fert A, Cros V, and Sampaio J. Skyrmions on the Track. Nat Nanotech (2013) 8:152-6. doi:10.1038/nnano.2013.29

2. Nagaosa N, and Tokura Y. Topological Properties and Dynamics of Magnetic Skyrmions. Nat Nanotech (2013) 8:899-911. doi:10.1038/nnano.2013.243

3. Iwasaki J, Mochizuki M, and Nagaosa N. Universal Current-Velocity Relation of Skyrmion Motion in Chiral Magnets. Nat Commun (2013) 4:1463. doi:10.1038/ncomms2442

4. Boulle O, Vogel J, Yang H, Pizzini S, de Souza Chaves D, Locatelli A, et al. Room-temperature Chiral Magnetic Skyrmions in Ultrathin Magnetic Nanostructures. Nat Nanotech (2016) 11:449-54. doi:10.1038/nnano.2015.315

5. Büttner F, Lemesh I, and Beach GSD. Theory of Isolated Magnetic Skyrmions: From Fundamentals to Room Temperature Applications. Sci Rep (2018) 8: 4464. doi:10.1038/s41598-018-22242-8

6. Yuan HY, and Wang XR. Skyrmion Creation and Manipulation by NanoSecond Current Pulses. Sci Rep (2016) 6:22638. doi:10.1038/srep22638

7. Silva RL, Silva RC, Pereira AR, and Moura-Melo WA. Antiferromagnetic Skyrmions Overcoming Obstacles in a Racetrack. J Phys Condens Matter (2019) 31:225802. doi:10.1088/1361-648x/ab0abd

8. Kim K-W, Moon K-W, Kerber N, Nothhelfer J, and Everschor-Sitte K. Asymmetric Skyrmion Hall Effect in Systems with a Hybrid Dzyaloshinskii-Moriya Interaction. Phys Rev B (2018) 97:224427. doi:10.1103/physrevb.97.224427

9. Bessarab PF, Yudin D, Gulevich DR, Wadley P, Titov M, and Tretiakov OA. Stability and Lifetime of Antiferromagnetic Skyrmions. Phys Rev B (2019) 99(R):140411. doi:10.1103/physrevb.99.140411

10. Liang JJ, Yu JH, Chen J, Qin MH, Zeng M, Lu XB, et al. Magnetic Field Gradient Driven Dynamics of Isolated Skyrmions and Antiskyrmions in Frustrated Magnets. New J Phys (2018) 20:053037. doi:10.1088/1367-2630/ aac24c

11. Barker J, and Tretiakov OA. Static and Dynamical Properties of Antiferromagnetic Skyrmions in the Presence of Applied Current and Temperature. Phys Rev Lett (2016) 116:147203. doi:10.1103/ physrevlett.116.147203

12. Zhang J, Zhang Y, Gao Y, Zhao G, Qiu L, Wang K, et al. Magnetic Skyrmions in a Hall Balance with Interfacial Canted Magnetizations. Adv Mater (2020) 32: 1907452. doi:10.1002/adma.201907452

13. Zhang X, Zhou Y, and Ezawa M. Antiferromagnetic Skyrmion: Stability, Creation and Manipulation. Sci Rep (2016) 6:24795. doi:10.1038/srep24795

14. Akosa C, Tretiakov O, Tatara G, and Manchon A. Theory of the Topological Spin Hall Effect in Antiferromagnetic Skyrmions: Impact on Current-

\section{AUTHOR CONTRIBUTIONS}

SG conceived the research project, SG and ZJ performed the computations. SG, YY, ZJ, TL, YL, and MQ commented the modeling and discussed the results. MQ and SG wrote the manuscript.

\section{FUNDING}

The work was supported by the Natural Science Foundation of China (Grant No. 51971096), the Science and Technology Planning Project of Guangzhou in China (Grant No. 201904010019), the Natural Science Foundation of Guangdong Province (Grant No. 2019A1515011028), Special Funds for the Cultivation of Guangdong College Students Scientific and Technological Innovation (Grant No. pdjh2020a0148), and National college students' innovation and entrepreneurship training program (Grant No. 202110574049).

Induced Motion. Phys Rev Lett (2018) 121:097204. doi:10.1103/ physrevlett.121.097204

15. Jin C, Song C, Wang J, and Liu Q. Dynamics of Antiferromagnetic Skyrmion Driven by the Spin Hall Effect. Appl Phys Lett (2016) 109:182404. doi:10.1063/ 1.4967006

16. Göbel B, Mook A, Henk J, and Mertig I. Antiferromagnetic Skyrmion Crystals: Generation, Topological Hall, and Topological Spin Hall Effect. Phys Rev B (2017) 96:060406(R). doi:10.1103/physrevb.96.060406

17. Buhl PM, Freimuth F, Blügel S, and Mokrousov Y. Topological Spin Hall Effect in Antiferromagnetic Skyrmions. Phys Status Solidi RRL (2017) 11:1700007. doi:10.1002/pssr.201700007

18. Yuan HY, Liu Q, Xia K, Yuan Z, and Wang XR. Proper Dissipative Torques in Antiferromagnetic Dynamics. EPL (2019) 126:67006. doi:10.1209/0295-5075/ 126/67006

19. Liang X, Zhao G, Shen L, Xia J, Zhao L, Zhang X, et al. Dynamics of an Antiferromagnetic Skyrmion in a Racetrack with a Defect. Phys Rev B (2019) 100:144439. doi:10.1103/physrevb.100.144439

20. Zhang X, Ezawa M, Xiao D, Zhao GP, Liu Y, and Zhou Y. All-magnetic Control of Skyrmions in Nanowires by a Spin Wave. Nanotechnology (2015) 26:225701. doi:10.1088/0957-4484/26/22/225701

21. Yu H, Xiao J, and Schultheiss H. Magnetic Texture Based Magnonics. Phys Rep (2021) 905:1-59. doi:10.1016/j.physrep.2020.12.004

22. Wang ZY, Yuan HY, Cao YS, Li ZX, Duine RA, and Yan P. Magnonic Frequency Comb through Nonlinear Magnon-Skyrmion Scattering. Phys Rev Lett (2021) 127:037202. doi:10.1103/physrevlett.127.037202

23. Iwasaki J, Beekman AJ, and Nagaosa N. Theory of Magnon-Skyrmion Scattering in Chiral Magnets. Phys Rev B (2014) 89:064412. doi:10.1103/ physrevb.89.064412

24. Shen M, Zhang Y, Ou-Yang J, Yang X, and You L. Motion of a Skyrmionium Driven by Spin Wave. Appl Phys Lett (2018) 112:062403. doi:10.1063/1.5010605

25. Proskurin I, Stamps RL, Ovchinnikov AS, and Kishine J-I. Spin-Wave Chirality and its Manifestations in Antiferromagnets. Phys Rev Lett (2017) 119:177202. doi:10.1103/physrevlett.119.177202

26. Daniels MW, Yu W, Cheng R, Xiao J, and Xiao D. Topological Spin Hall Effects and Tunable Skyrmion Hall Effects in Uniaxial Antiferromagnetic Insulators. Phys Rev B (2019) 99:224433. doi:10.1103/physrevb.99.224433

27. Jin Z, Meng CY, Liu TT, Chen DY, Fan Z, Zeng M, et al. Magnon-driven Skyrmion Dynamics in Antiferromagnets: Effect of Magnon Polarization. Phys Rev B (2021) 104:054419. doi:10.1103/physrevb.104.054419

28. Pickart SJ, Collins MF, and Windsor CG. Spin-Wave Dispersion in KMnF3. J Appl Phys (1966) 37:1054-5. doi:10.1063/1.1708332

29. Heeger AJ. Spin-Wave Instability and Premature Saturation in Antiferromagnetic Resonance. Phys Rev (1963) 131:608-16. doi:10.1103/physrev.131.608 
30. Qaiumzadeh A, Kristiansen LA, and Brataas A. Controlling Chiral Domain Walls in Antiferromagnets Using Spin-Wave Helicity. Phys Rev B (2018) 97: 020402(R). doi:10.1103/physrevb.97.020402

31. Saiki K. Resonance Behaviour in Canted Antiferromagnet KMnF3. J Phys Soc Jpn (1972) 33:1284-91. doi:10.1143/jpsj.33.1284

32. Lan J, Yu W, and Xiao J. Antiferromagnetic Domain wall as Spin Wave Polarizer and Retarder. Nat Commun (2017) 8:178. doi:10.1038/s41467-017-00265-5

33. Jin Z, Liu T, Li W, Zhang X, Hou Z, Chen D, et al. Dynamics of Antiferromagnetic Skyrmions in the Absence or Presence of Pinning Defects. Phys Rev B (2020) 102:054419. doi:10.1103/physrevb.102.054419

34. Wang XS, Yuan HY, and Wang XR. A Theory on Skyrmion Size. Commun Phys (2018) 1:31. doi:10.1038/s42005-018-0029-0

35. Schütte C, and Garst M. Magnon-skyrmion Scattering in Chiral Magnets. Phys Rev B (2014) 90:094423. doi:10.1103/physrevb.90.094423

36. Tveten EG, Qaiumzadeh A, Tretiakov OA, and Brataas A. Staggered Dynamics in Antiferromagnets by Collective Coordinates. Phys Rev Lett (2013) 110: 127208. doi:10.1103/physrevlett.110.127208

37. Legrand W, Maccariello D, Ajejas F, Collin S, Vecchiola A, Bouzehouane K, et al. Room-temperature Stabilization of Antiferromagnetic Skyrmions in Synthetic Antiferromagnets. Nat Mater (2020) 19:34-42. doi:10.1038/ s41563-019-0468-3

38. Cheng R, Daniels MW, Zhu J-G, and Xiao D. Antiferromagnetic Spin Wave Field-Effect Transistor. Sci Rep (2016) 6:24223. doi:10.1038/srep24223
39. Cheng R, Xiao J, Niu Q, and Brataas A. Spin Pumping and Spin-Transfer Torques in Antiferromagnets. Phys Rev Lett (2014) 113:057601. doi:10.1103/ physrevlett.113.057601

40. Cheng R, and Niu Q. Dynamics of Antiferromagnets Driven by Spin Current. Phys Rev B (2014) 89:081105(R). doi:10.1103/physrevb.89.081105

Conflict of Interest: The authors declare that the research was conducted in the absence of any commercial or financial relationships that could be construed as a potential conflict of interest.

Publisher's Note: All claims expressed in this article are solely those of the authors and do not necessarily represent those of their affiliated organizations, or those of the publisher, the editors, and the reviewers. Any product that may be evaluated in this article, or claim that may be made by its manufacturer, is not guaranteed or endorsed by the publisher.

Copyright (c) 2021 Guan, Yang, Jin, Liu, Liu and Qin. This is an open-access article distributed under the terms of the Creative Commons Attribution License (CC BY). The use, distribution or reproduction in other forums is permitted, provided the original author(s) and the copyright owner(s) are credited and that the original publication in this journal is cited, in accordance with accepted academic practice. No use, distribution or reproduction is permitted which does not comply with these terms. 